\title{
Physical activity, undernutrition and child development
}

\author{
BY J. M. MEEKS GARDNER AND \\ S. M. GRANTHAM-MCGREGOR \\ Tropical Metabolism Research Unit, University of the West Indies, Mona, Kingston 7, Jamaica, \\ West Indies
}

\begin{abstract}
Although evidence is accumulating which suggests a causal relationship between undernutrition and poor development in young children (Simeon \& GranthamMcGregor, 1990; Pollitt et al. 1993), the mechanism linking the two remains unclear. One hypothesis is that lack of dietary energy leads to reduced activity levels, which in turn leads to reduced exploration and subsequently results in developmental delays (Levitsky, 1979). This is known as the 'functional isolation hypothesis'. In the present review we will discuss the evidence for this hypothesis in young children as it relates to activity levels.
\end{abstract}

\section{ACTIVITY LEVELS OF UNDERNOURISHED CHILDREN}

The foundation of the functional isolation hypothesis is that undernourished children have reduced activity levels. Early investigators described reduced activity in severely undernourished children, but systematic observations were not done (Williams, 1935; Geber \& Dean, 1956). In Jamaica, the behaviour of seventeen severely malnourished children in the acute stage in hospital was compared with that of fourteen adequately nourished children who were hospitalized with other diseases, using systematic observations (Grantham-McGregor et al. 1991b). The malnourished children were significantly less active on admission to hospital but caught up to the controls 1 week later.

Few studies have been carried out on activity levels of mild to moderately undernourished children, and most of these have serious flaws in their study design. They can be divided into cross-sectional studies (Table 1), nutritional supplementation studies (Table 2), and studies involving the reduction of energy intake.

\section{Cross-sectional studies}

There are reports of activity in young undernourished children from three cross-sectional studies. In a pioneering study in Uganda, Rutishauser \& Whitehead (1972) compared the activity levels of twenty undernourished African children and five adequately nourished European children aged 1.5-5 years using time-and-motion techniques. The African children spent significantly more time than the European children sitting and standing, and less time being carried, walking or running. However, the control group was small, and had a wide age-range. Further, the controls were of different ethnic and class backgrounds, which may affect activity patterns and, thus, were inappropriate comparisons for the African children; therefore it is difficult to interpret the findings. The activity patterns of eighty-one Gambian and fifty-three British children aged 6,12 or 18 months were compared in another study using a combination of diary records and direct observations (Lawrence et al. 1991). The mean weights-for-age of the Gambian children were lower than those of the UK children at each time-point. 
Table 1. Cross-sectional studies of activity levels in undernourished children

\begin{tabular}{|c|c|c|c|}
\hline & & \multicolumn{2}{|c|}{$\begin{array}{l}\text { Differences between under- } \\
\text { nourished and adequately } \\
\text { nourished children from }\end{array}$} \\
\hline & & Same culture & Different culture \\
\hline \multicolumn{4}{|l|}{ Preschool children } \\
\hline Rutishauser \& Whitehead (1972) & Uganda & - & + \\
\hline Lawrence et al. (1991) & Gambia & $=$ & + \\
\hline Torun $(1990)$ & Guatemala & + & - \\
\hline \multicolumn{4}{|l|}{ School-aged children } \\
\hline Spurr et al. $(1986,1988)$ & Colombia & $=$ & -- \\
\hline
\end{tabular}

,- Not done; $=$, no differences; + , undernourished children less active.

Table 2. Studies of activity levels in supplemented undernourished children

\begin{tabular}{llll}
\hline & & $\begin{array}{c}\text { Activity } \\
\text { response to } \\
\text { supplementation }\end{array}$ & Comment \\
\hline $\begin{array}{l}\text { Preschool children } \\
\text { Torun (1990) }\end{array}$ & $\begin{array}{l}\text { Guatemala } \\
\text { Colombia }\end{array}$ & + & $\begin{array}{c}\text { No non-supplemented group } \\
\text { Act al. }(1981)\end{array}$ \\
$\begin{array}{l}\text { Chavez et al. }(1972) \\
\text { Mexico }\end{array}$ & + & $\begin{array}{c}\text { Non-supplemented group 1 year } \\
\text { earlier }\end{array}$ \\
Barrett \& Radke-Yarrow (1985) & Guatemala & + & $\begin{array}{c}\text { Supplemented group was } \\
\text { self-selected }\end{array}$ \\
Spurr \& Reina (1987) & Colombia & - & \\
\hline \hline
\end{tabular}

+ , Activity increased with supplementation; ?, response of activity uncertain; - , no increase in activity with supplementation.

The Gambian children spent significantly less time crawling, walking or running, and more time sitting than the UK children. However, because there were vast cultural differences between the UK and Gambian children, and different climates and methodologies used, the authors did not ascribe the differences in activity patterns to differences in the nutritional status of the groups. More importantly, the Gambian children of differing nutritional status were also compared. Those above $80 \%$ weight-forage at 6 months were found to play with objects or other people for longer than those with weights-for-age less than $80 \%$. However, there were no significant differences in the time spent in the different levels of activities. These results are more valid than those between the UK and Gambian children for assessing differences resulting from undernutrition.

In Guatemala, sixty-nine poor children aged 2-6 years old were observed on two week-days between 08.00 and 17.00 hours (Torun, 1990). Activities were recorded using time-and-motion techniques similar to those used by Rutishauser \& Whitehead (1972). 
Mildly malnourished children, $81-90 \%$ of the NCHS references ( $n 21$ ), spent more time in sedentary activities and less time in more vigorous activities than the adequately nourished children (above 90\% NCHS; $n$ 43). However, it is difficult to evaluate this study because details have not yet been published.

In studies on underprivileged Colombian schoolchildren, differences between the energy spent in activities of marginally undernourished school-aged children and controls were ascribed to differences in lean body mass (Spurr et al. 1986; Spurr \& Reina, 1988).

Conclusions from cross-sectional studies. Of the studies reviewed, conclusions cannot be drawn from those by Rutishauser \& Whitehead (1972), while Lawrence et al. (1991) and Spurr et al. (1986) and Spurr \& Reina (1988) did not detect reduced activity levels in undernourished children. Torun (1990) was the only investigator to find that the undernourished children had reduced activity; however, details of that study have not been reported. Thus, there is very limited evidence of reduced activity in undernourished children in their usual environment.

\section{Supplementation studies}

The response of children's activity levels to supplementation was examined in four studies.

In the Guatemalan study described by Torun (1990), sixty of the children were supplemented for 4 months and observed again. Children whose weight-for-height improved spent less time in sedentary activities than those whose weight-for-height remained the same or decreased. However, it is difficult to evaluate this study because details have not yet been published and there was no non-supplemented group. Factors such as age, sex, and initial nutritional status may account for some of the changes.

In Colombia (Super et al. 1981), poor families were assigned to one of six groups of which four groups will be discussed: a group receiving a supplement ( $n$ 82), a group given a maternal education programme ( $n 58)$, a group given a supplement and a maternal education programme ( $n$ 58), and a control group receiving neither intervention (controls, $n 90$ ). At 4 and 8 months of age the children were observed and gross body movements were recorded. However, this variable was only reported as part of a factor which included other behaviours. Supplemented children had a higher score on this factor at 4 months, but the effect of the interventions on activity alone cannot be determined. At 8 months, gross body movements were not included in the factor.

Pregnant women and children under 7 years were offered a nutritional supplement on a self-selection basis in four villages in Guatemala (Barrett \& Radke-Yarrow, 1985). Children who received the most supplement up to 4 years old (high-supplement group) were compared with those who received less supplementation (low-supplement group). Children from three of the villages ( $n$ 138) were observed at 6-8 years old in free play and structured activities. The high-supplement group had higher rates of moderate and very high activity levels than the low-supplement group. However, it is unclear whether some of the children were still receiving supplement. Also, self-selection could be a severe confounder in this study in that there is likely to have been pre-existing differences between those who selected high and low levels of supplement.

In another study the physical activity of seventeen non-supplemented children was compared with that of nineteen supplemented children from the same village in Guatemala born 1 year later (Chavez et al. 1972). When the children were between 4 and 
12 months old they were observed in their homes, and the number of foot contacts with the supporting surface was recorded. The supplemented children showed a significantly higher activity index from 13 months of age, which steadily increased up to 24 months.

Unfortunately, there are several problems with this study design. The number of children in parts of the age-range must have been very few. Because of the time lag between the groups, the differences may have resulted from factors other than the supplementation. There was no control for the increased attention received by the supplemented children (Grantham-McGregor et al. 1990) and the attitudes of the mothers and other family members may have been affected by the supplementation programme, which may in turn have affected the children's behaviour.

Spurr \& Reina (1987) examined the effect of nutritional supplementation on the energy expended in activity in undernourished Colombian girls aged 8-11 years. After more than 4 months no increase was found in spite of an increase in growth velocity.

Conclusions from supplementation studies. Two studies cannot be evaluated because in one the activity data were not presented separately (Super et al. 1981), and in the other no details were provided (Torun, 1990). In a further two studies interpretations cannot be drawn because of the problems with study design (Barrett \& Radke-Yarrow, 1985 and Chavez et al. 1982). In the fifth study (Spurr \& Reina, 1987) there was no activity response to supplementation. However, these children may be somewhat old to test the functional isolation hypothesis. The activity response to supplementation, therefore, is not established.

\section{Studies of activity with reduced energy intake}

Two studies were carried out at a clinical centre in Guatemala on boys who had recovered from severe malnutrition. In one, five boys aged $1.5-4.5$ years were studied for $4 \mathrm{~d}$ on a high-energy diet and for $4 \mathrm{~d}$ on a low-energy diet (Viteri \& Torun, 1981). Observations were done using minute-by-minute time-and-motion techniques. After the reduction in energy intake the time spent in more energy-demanding activities was reduced.

In the other study, five boys aged 22-40 months (Torun \& Viteri, 1981) were studied over three $40 \mathrm{~d}$ periods when they received three different levels of dietary energy. The boys spent more energy in physical activity, as determined by heart-rate monitoring, at the highest level of energy intake, but there was no difference at the two lower levels.

Conclusions from studies with reduced energy intakes. Both these studies suffer from small sample sizes and it is uncertain whether the response to reduced intake can be extrapolated to predict the behaviour of undernourished children in the everyday situation. In addition, the psychological effect of reducing intakes in previously severely malnourished children cannot be ignored.

\section{ACTIVITY AND DEVELOPMENT}

From the functional isolation hypothesis, it would be expected that reduced activity would precede and predict poor developmental levels. Two studies have included measures of both activity and development in undernourished children.

Nutritional supplementation was associated with an increase in the children's activity levels and an improvement in development in Mexican (Chavez et al. 1972; Chavez \& Martinez, 1982) and Guatemalan studies (Barrett \& Radke-Yarrow, 1985). However, 


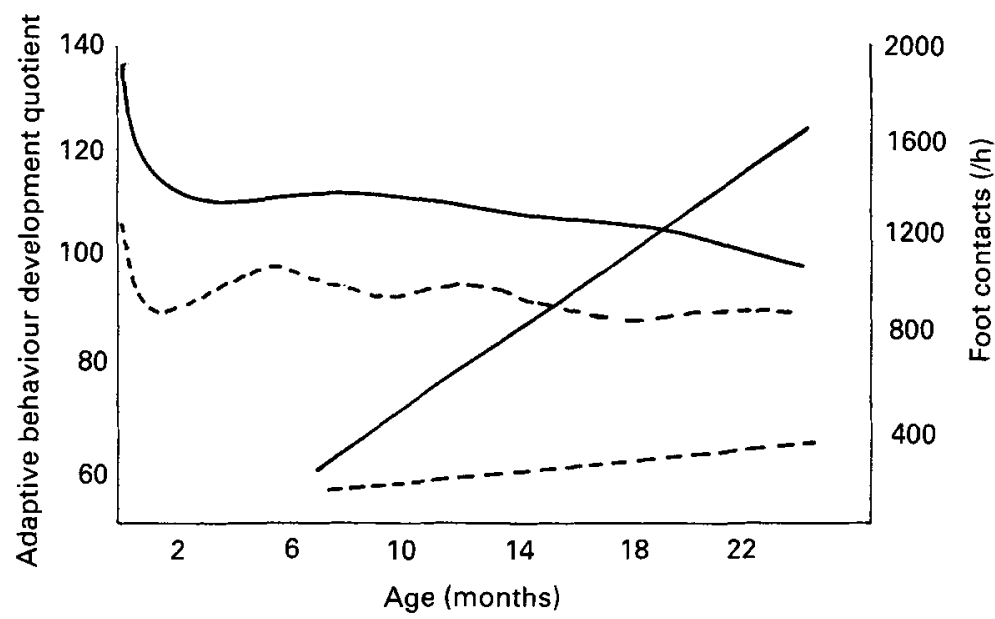

Fig. 1. Activity ratings and scores on the adaptive behaviour subscale (Gessel) in two groups of children: ( - ), supplemented; (- - ), non-supplemented. (From Chavez \& Martinez, 1982. Reprinted with permission from IDECG.)



Fig. 2. Activity ratings and scores on the motor subscale (Gessel) in two groups of children: (-), supplemented: (- - ), non-supplemented. (From Chavez \& Martinez, 1982. Reprinted with permission from IDECG.)

there was no attempt to link activity to development. In the Mexican study, improvement in development preceded the increase in activity rather than the other way around (Grantham-McGregor et al. 1990; Figs. 1 and 2); therefore, there is no evidence that reduced activity in undernourished children is causally related to poor development.

\section{The Jamaican study}

We carried out a study in Jamaica in order to investigate the relationship between activity and development in growth-retarded (stunted) children. This was part of a larger 
study of the effects of nutritional supplementation with or without psychosocial stimulation on development and growth of stunted children (Grantham-McGregor et al. 1991a; Walker et al. 1991).

Stunting, which was not specifically looked at in any of the studies reviewed previously, was chosen as the index of undernutrition since it is extremely prevalent, affecting up to $40 \%$ of children in the developing world (Keller, 1988). Furthermore, it has been demonstrated in many studies that stunting is associated with poor development (Simeon \& Grantham-McGregor, 1990).

Seventy-eight stunted children (weight-for-height more than 2 SD below NCHS reference; Hamill et al. 1977), aged 12-24 months, were randomly assigned to one of three treatment groups: one group receiving nutritional supplementation, another receiving both supplementation and psychosocial stimulation ('both'), and controls who received neither intervention. Twenty-six matched non-stunted children were also studied. For the purposes of the present report we will focus on the effect of the supplement.

Activity levels were measured by extensive observations in the homes. In the observation method used by Rutishauser \& Whitehead (1972) and Torun (1990), any activity which occurred throughout every $10 \mathrm{~min}$ period was recorded. The time spent on each activity was estimated by dividing the $10 \mathrm{~min}$ period by the number of recorded activities. We reduced the $10 \mathrm{~min}$ observation periods to $1 \mathrm{~min}$, which more closely approximates the actual timing of the observations (Meeks Gardner et al. 1989). An activity score was calculated by adding values for the percentage time spent in each activity multiplied by the estimated energy cost of that activity (Torun, 1990). This score was highly reliable over several days.

The children's development was assessed on enrolment and every 6 months using four subscales of the Griffith's Mental Development Scales. Developmental quotients (DQ) were calculated by averaging the four subscales scores. On enrolment the stunted children had poorer levels of development than the non-stunted children, but with supplementation over 2 years the deficit in their DQ was reduced (Grantham-McGregor et al. 1991a).

On enrolment the stunted children spent significantly more time in light activities, and significantly less time in moderate or vigorous activities. Their activity score was also significantly lower. However, after 6 months the activity levels of the stunted children had caught up to those of the non-stunted comparison children regardless of whether they received the supplement or not.

The lack of a supplementation effect on activity was unlikely to be due to inadequate intakes of the supplement since there was a significant growth response (Walker et al. 1991). On enrolment, activity levels made a significant contribution to the variance in the locomotor subscale only. After 6 months, activity levels were no longer significantly related to any of the subscales.

Conclusions from the Jamaican study. We found reduced activity levels of stunted children at one age but not at another. This may help to explain the different findings in other studies.

In Jamaica, activity was related to locomotor development only, but only at the earlier stage, not the later stage. We hypothesized that reduced activity was more likely to be a result of delayed locomotor development rather than reduced activity predicting poor development. It is possible that locomotor ability predicts activity levels up to the stage 
when certain skills are acquired, but not subsequently. For instance, on enrolment a much greater proportion of the non-stunted than the stunted children could run, but all children could run 6 months later. Running may be a critical skill in determining activity levels.

Supplementation improved development but did not affect activity, and the control stunted group continued to have poor developmental levels in spite of increasing activity levels.

These findings only partly support the functional isolation hypothesis as far as stunted children are concerned. It is likely that the quality of the child's exploration and other characteristics such as curiosity and alertness may be more important than gross motor activity in influencing development.

The Jamaican work was supported by the Ford Foundation and the United Nations University.

\section{REFERENCES}

Barrett, D. E. \& Radke-Yarrow, M. (1985). Effects of nutritional supplementation on children's responses to novel frustrations and competitive situations. American Journal of Clinical Nutrition 42, 102-120.

Chavez, A. \& Martinez, C. (1982). Growing Up in a Developing Community. Guatemala: INCAP.

Chavez, A., Martinez, C. \& Bourges, H. (1972). Nutrition and development of infants from poor rural areas 2. Nutritional level and physical activity. Nutrition Reports International 5, 139-144.

Geber, M. \& Dean, R. F. (1956). The psychological changes accompanying kwashiorkor. Courrier du CNRS 6, 6-15.

Grantham-McGregor, S., Meeks Gardner, J., Walker, S. \& Powell, C. (1990). The relationship between undernutrition, activity levels and development in young children. In Activity, Energy Expenditure and Energy Requirements of Infants and Children, pp. 361-384 [B. Schurch and N. Scrimshaw, editors]. Lausanne: IDECG/Nestlé Foundation.

Grantham-McGregor, S. M., Powell, C. A., Walker, S. P. \& Himes, J. H. (1991a). Nutritional supplementation, psychosocial stimulation and development of stunted children: The Jamaican study. Lancet 338, 1-5.

Grantham-McGregor, S., Stewart, M. \& Powell, C. (1991b). Behaviour of severely malnourished children in a Jamaican hospital. Developmental Medicine \& Child Neurology 33, 706-714.

Hamill, P. V. V., Drizol, T. A., Johnson, C. L., Reed, R. B. \& Rock, A. F. (1977). Growth curves for children, birth-18 years. Vital and Health Statistics series vol. II, no. 165. (CDHEW publication no. 78) Hyatsville, MD, USA: National Centre for Health Statistics.

Keller, W. (1988). The epidemiology of stunting. In Linear Growth Retardation in Less Developed Countries. Nestlé Nutrition Workshop no. 14, pp. 17-40 [J. C. Waterlow, editor]. New York: Raven Press.

Lawrence, M., Lawrence, F., Durnin, J. V. G. A. \& Whitehead, R. G. (1991). A comparison of physical activity in Gambian and UK children aged 6-18 months. European Journal of Clinical Nutrition 45, 243-252.

Levitsky, D. A. (1979). Malnutrition and hunger to learn. In Malnutrition, Environment and Behaviour [D. A. Levitsky, editor]. Ithaca: Cornell University Press.

Meeks Gardner, J. M., Grantham-McGregor, S. M., Chang, S. M. \& Powell, C. A. (1990). Dietary intake and observed activity of stunted and non-stunted children in Kingston, Jamaica. Part II: Observed activity. European Journal of Clinical Nutrition 44, 585-593.

Pollitt, E., Gorman, K., Engle, P., Martorell, R. \& Rivera, J. (1993). Early supplementary feeding and cognition: Effects over two decades. Monographs of the Society for Research in Child Development no. 235.

Rutishauser, I. E. \& Whitehead, R. G. (1972). Energy intake and expenditure in 1-3-year-old Ugandan children living in a rural environment. British Journal of Nutrition 28, 145-152.

Simeon, D. \& Grantham-McGregor, S. (1990). Nutritional deficiencies and children's behaviour and development. Nutrition Research Reviews 3, 1-24.

Spurr, G. B. \& Reina, J. C. (1987). Marginal malnutrition in school-aged Colombian girls: Dietary interventions and daily energy expenditure. Human Nutrition: Clinical Nutrition 41C, 93-104. 
Spurr, G. B. \& Reina, J. C. (1988). Patterns of daily energy expenditure in normal and marginally undernourished school-aged Colombian children. European Journal of Clinical Nutrition 42, 819-834.

Spurr, G. B., Reina, J. C. \& Barac-Nieto, M. (1986). Marginal malnutrition in school-aged Colombian boys: metabolic rate and estimated daily energy expenditure. American Journal of Clinical Nutrition 44, 113-126.

Super, C. M., Clement, J., Vuori, L., Christiansen, N., Mora, J. O. \& Herrera, M. G. (1981). Infant and caretaker behaviour as mediators of nutritional and social intervention in the barrios of Bogota. In Culture and Early Intervention, pp. 171-188 [T. Feildt, A. Sostek, P. Vietze and P. Leiderman, editors]. Hillsdale NJ: Earlbaum.

Torun, B. (1990). Short- and long-term effects of low or restricted energy intakes on the activity of infants and children. In Activity, Energy Expenditure and Energy Requirements of Infants and Children, pp. 335-358 [B. Schurch and N. S. Scrimshaw, editors]. Lausanne: IDECG/Nestlé Foundation.

Torun, B. \& Viteri, F. E. (1981). Energy requirements of pre-school children and effects of varying energy intakes on protein metabolism. In Protein-Energy Requirements of Developing Countries: Evaluation of New Data. UNU Food and Nutrition Bulletin, Suppl. 5, pp. 229-241 [B. Torun, V. R. Young and W. M. Rand, editors].

Viteri, F. E. \& Torun, B. (1981). Nutrition, physical activity and growth. In The Biology of Normal Human Growth, pp. 265-273 [M. Ritzen, A. Aperia, K. Hall, A. Larsson, A. Zetterberg and R. Zetterstrom, editors]. New York: Raven Press.

Walker, S., Powell, C., Grantham-McGregor, S., Himes, J. \& Chang, S. (1991). Nutritional supplementation, psychosocial stimulation and growth of stunted children: the Jamaican Study. American Journal of Clinical Nutrition 54, 642-648.

Williams, C. D. (1935). Kwashiorkor. Lancet ii, 1151-1152. 